\title{
Akustisches Monitoring \\ von Rauhautfledermaus \\ an Windenergieanlagen: Ist ein \\ zweites Ultraschallmikrofon am \\ Turm notwendig?
}

\section{Acoustic monitoring of Nathusius' pipistrelles (Pipistrellus nathusii): Is a second ultrasonic microphone at the tower needed?}

\section{Lothar Bach, Petra Bach und Raimund Kesel}

\section{Zusammenfassung}

Energie aus Windkraft spielt im Rahmen der Förderung erneuerbarer Energieträger eine immer größere Rolle, allerdings kollidieren Fledermäuse regelmäßig mit Windenergieanlagen (WEA). Um derartige Kollision zu verhindern, fordern Behörden im Rahmen von Genehmigungsverfahren die Einführung von Abschaltzeiten, zumeist unter Zuhilfenahme des Programms ProBat. Im küstennahen Umfeld bzw. in Bereichen mit hoher Aktivität an Rauhautfledermäusen (Pipistrellus nathusii) wird dieses Programm bislang nur begrenzt eingesetzt. In Nordwestdeutschland wurde von uns in den letzten Jahren an 20 WEA zusätzlich ein zweites Mikrofon am Turm etwa 10-15 m unterhalb des tiefsten Streifpunkts der Rotorblätter getestet (Turmmikrofon). Auf dieser Höhe lässt sich

L. Bach $(\bowtie) \cdot$ P. Bach

Freilandforschung, zoologische Gutachten, Bremen, Deutschland

E-Mail: lotharbach@bach-freilandforschung.de

P. Bach

E-Mail: petrabach@ bach-freilandforschung.de

R. Kesel

ecosurvey, Bremen, Deutschland

E-Mail:kesel@ecosurvey.de 
eine deutlich höhere akustische Aktivität der Rauhautfledermaus messen als auf Gondelhöhe (Gondelmikrofon). Der akustische Erfassungsbereich beider Mikrofone überlappte sich hinsichtlich der Rauhautfledermaus nicht. Am Turmmikrofon zeigte sich nicht nur eine höhere Gesamtaktivität von Fledermäusen bzw. eine höhere Aktivität der Rauhautfledermaus, sondern auch eine unterschiedliche saisonale und nächtliche Verteilung der Aktivitäten im Vergleich zum Gondelmikrofon. Auch wenn das Aktivitätsmaximum am Turmmikrofon bei niedrigeren Windgeschwindigkeiten lag als beim Gondelmikrofon, war am Turmmikrofon die Aktivität noch etwa siebenmal höher als auf Nabenhöhe. Dies lag an der insgesamt höheren Aktivität von Fledermäusen am Turmmikrofon im Vergleich zum Gondelmikrofon; selbst bei relativ hohen Windgeschwindigkeiten $(\geq 8,0 \mathrm{~m} / \mathrm{s})$. Die relativ hohe akustische Aktivität von Rauhautfledermäusen am Turmmikrofon könnte erklären, warum Schlagopfer (speziell von Rauhautfledermäusen) ohne entsprechende akustische Aktivitäten in Gondelhöhe anfallen. Die kombinierte Nutzung von Mikrofonen am Turm und im Gondelbereich könnte die Entwicklung von Abschaltalgorithmen aus Sicht des Fledermausschutzes verbessern.

\section{Summary}

Wind energy is a major contributor of renewable energy, yet bats collide regularly with wind turbines (WT). To prevent such collisions, authorities of many federal states in Germany require the implementation of curtailment measures, most often by applying the ProBat-tool. Thus far, this tool has not been widely applied in coastal areas and in areas with high activity of Nathusius' pipistrelles (Pipistrellus nathusii). In NW-Germany, we surveyed 20 wind turbines for one or two years (total 29 WT-years). In addition to a microphone at nacelle height (nacelle microphone), we installed a second microphone about $10-15 \mathrm{~m}$ below the lowest operating range of the rotor blades (tower microphone). We measured a higher acoustic activity of bats at the tower microphone than at the nacelle microphone. The acoustic detection range of both microphones did not overlap for Nathusius' pipistrelles. The results show that the overall activity of bats as well as the acoustic activity of Nathusius' pipistrelles was higher at the tower microphone compared to the nacelle microphone. Moreover, the seasonal and nocturnal phenology was different between the two microphones. Even though peak activity of bats at the tower microphone occurred at lower wind speeds than at the nacelle microphone, overall bat activity was still seven times higher at the tower microphone compared with the nacelle microphone; even at relatively high wind speeds of $\geq 8,0 \mathrm{~m} / \mathrm{s}$. The relatively high acoustic activity of bats at the 
tower microphone may explain why fatalities (especially those of Nathusius' pipistrelles) occur without acoustic activity at the nacelle microphone. The combined use of microphones at nacelle height and at the tower could improve the development of bat friendly curtailments.

\subsection{Einleitung}

Die Erzeugung von Energie aus Windkraft spielt im Rahmen der nationalen Strategien zur Förderung erneuerbarer Energieträger und zur Abkehr von fossilen Brennstoffen und Kernenergie eine immer größer werdende Rolle. Laut Bundesverband Windenergie (BWE 2018) existieren in Deutschland etwa 30.518 WEA, welche 59.313 MW Energie produzieren. Infolge der zunehmenden Zahl an WEA treten vermehrt Probleme mit dem Artenschutz, vor allem beim Schutz von größeren Vögeln, von Fledermäusen und möglicherweise auch von Insekten, auf (Corten und Veldkamp 2001; Bernardino et al. 2013; Voigt et al. 2015; O'Shea et al. 2016; Frick et al. 2017; Behr et al. 2018; Lindemann et al. 2018). Um kollisionsgefährdete Tierarten an WEA zu schützen, müssen im Rahmen von Windkraftvorhaben wirksame Vermeidungsmaßnahmen erarbeitet werden. Neben der eigentlichen Standortwahl spielt für den Schutz von Fledermäusen die Beauflagung von Abschaltzeiten als wirksamste Vermeidungsmaßnahme eine wichtige Rolle (Arnett und May 2016; Voigt et al. 2015; Rodrigues et al. 2015). Dem Ziel der Entwicklung wirksamer Abschaltalgorithmen hat sich seit Jahren das vom Bundesministerium für Umwelt, Naturschutz und Reaktorsicherheit und dem Bundesministerium für Wirtschaft und Energie geförderte Projekt RENEBAT verschrieben (Brinkmann et al. 2011; Behr et al. 2015, 2018). Im Rahmen dieses Projekts wurde methodisch und einheitlich bundesweit eine Anzahl an WEA intensiv auf die Beziehung von Fledermausaktivität, welche über automatisierte Ultraschalldetektorsysteme in Gondelhöhe ermittelt wurde, und der Anzahl von geschlagenen Fledermäusen untersucht. Neben einem großen Erkenntnisgewinn über die Interaktion von Fledermäusen mit WEA wurde eine Berechnungsmethode (nachfolgend als ProBat bezeichnet) entwickelt, welche es ermöglichen soll, aus der akustischen Erfassung von Fledermäusen in Gondelhöhe unter Berücksichtigung von Umweltvariablen wie der Windgeschwindigkeit einen Abschaltmodus abzuleiten, der die Schlagopferzahlen gemäß dem Tötungsverbot nach §44 (1) Satz 1 BNatSchG unter eine kritische Grenze senken soll.

Neben diversen methodischen Problemen hinsichtlich der zunehmenden Länge der Rotoren und der höheren Positionierung der Gondeln (Lindemann et al. 2018) tritt für die küstennahen Gebiete und möglicherweise auch für weitere Standorte ein zusätzliches Problem auf. Eine hohe Flugaktivität speziell von Rauhautfledermäusen (Pipistrellus nathusii), einer Art mit hohem Kollisionsrisiko, wurde in der Vergangenheit über die Berechnungsmethode von ProBat 
nur unzureichend abgebildet (Behr et al. 2015). Der Grund hierfür scheint zum einen darin zu liegen, dass bei den bundesweiten Untersuchungen im Rahmen des RENEBAT-Vorhabens vornehmlich küstenferne Standorte untersucht wurden, wo möglicherweise die Migration der Rauhautfledermäuse von geringerer Bedeutung ist als an küstennahen Standorten. Außerdem setzte die Berechnungsmethode von ProBat bis zur rezenten Version 6.1 (Erscheinungsjahr 2019) eine Mindestgesamtaktivität auf Nabenhöhe von 150 akustischen Aufnahmen voraus, die häufig in Gondelhöhe von WEA direkt an der Küste nicht erreicht wird. Trotzdem werden an solchen Standorten oftmals tote Rauhautfledermäuse gefunden (Kap. 4), d. h., eine ausschließlich akustische Erfassung der Rauhautfledermaus auf Gondelhöhe, wie sie der Niedersächsische Winderlass einfordert (NMU 2016), unterschätzt vermutlich die tatsächliche Zahl gefundener Schlagopfer an küstennahen Standorten. Aus diesem Grund wurde 2014 sowohl von EUROBATS (Rodrigues et al. 2015) als auch von Seiten verschiedener Gutachter und Behörden (z. B. Landkreis Aurich) überlegt, ob ein zweites Mikrofon, welches am Turm knapp unterhalb des tiefsten Streifradius der Rotorspitzen angebracht wird, möglicherweise eine bessere Grundlage zur Abschätzung des Schlagrisikos von Rauhautfledermäusen ermöglichen würde. Um dies zu überprüfen wurden bei Monitorings im Rahmen von Genehmigungsverfahren testweise Turmmikrofone eingesetzt. Das Ziel dieses Kapitels ist es, verschiedene Untersuchungen hierzu aus den letzten drei Jahren zusammenzutragen, auszuwerten und zu diskutieren.

\subsection{Methode}

\subsubsection{Studiengebiet und Untersuchungsaufbau}

Im Rahmen unserer Studie werteten wir Untersuchungen aus den Jahren 2016 bis 2018 aus dem nordwestdeutschen Küstenraum aus. Alle untersuchten Windparks (Tab. 5.1) lagen in den Landkreisen Aurich und Wittmund (Nordwest-Niedersachsen, Ostfriesland). Außer dem Windpark S standen alle WEA im Offenland mit großen Abständen zu Gehölzen. Im Windpark S standen drei der fünf WEA an Hecken, wobei die Rotorspitze sich den Baumwipfeln auf etwa 70-80 m annäherte. An allen untersuchten WEA wurde im Turmfuß ein Avisoft-System (Avisoft Bioacoustics, Berlin) mit zwei Mikrofonausgängen angebracht. Beide Mikrofone wurden über entsprechend lange Kabel mit dem Avisoft-Gerät verbunden. Das erste Mikrofon (nachfolgend Gondelmikrofon genannt) wurde entsprechend gängigen Empfehlungen im hinteren Bereich der Gondel vertikal nach unten zeigend ausgerichtet. Das zweite Mikrofon (nachfolgend Turmmikrofon genannt) bestand aus einem Stabmikrofon, welches durch eine Öffnung mit einem Durchmesser von $13 \mathrm{~mm}$ im Turm horizontal ausgerichtet eingebaut wurde. Dieses Mikrofon wurde etwa 10-15 m (Tab. 5.1) 
Tab. 5.1 Untersuchte WEA-Typen in verschiedenen Windparks, Nabenhöhen und Rotorlängen sowie Positionshöhe des Turmmikrofons und die Anzahl der Untersuchungsjahre

Tab. 5.1 Studied types of wind turbines in different wind farms, nacelle heights, rotor blade length, position oft the tower microphone and number of study years

\begin{tabular}{l|l|l|l|l|l|l}
\hline Windpark & $\begin{array}{l}\text { Anzahl } \\
\text { WEA }\end{array}$ & WEA-Typ & $\begin{array}{l}\text { Nabenhöhe } \\
(\mathrm{m})\end{array}$ & $\begin{array}{l}\text { Rotorradius } \\
(\mathrm{m})\end{array}$ & $\begin{array}{l}\text { Höhe Turm- } \\
\text { mikrofon } \\
(\mathrm{m})\end{array}$ & $\begin{array}{l}\text { Untersuchte } \\
\text { Jahre* }\end{array}$ \\
\hline $\mathrm{H}$ & 1 & E-70/E4 & 113,5 & 35 & 64 & 2 \\
\hline $\mathrm{T}$ & 5 & E 115 & 135,4 & 58 & 65 & 1 \\
\hline $\mathrm{S}$ & 5 & E 115 & 135,4 & 58 & 65 & 2 \\
\hline $\mathrm{G}$ & $4(3)$ & E 101 & 135,4 & 50 & 75 & 2 \\
\hline $\mathrm{U}$ & 3 & E 115 & 135,4 & 58 & 65 & 1 \\
\hline $\mathrm{W}$ & 2 & E 115 & 135,4 & 58 & 65 & 1 \\
\hline
\end{tabular}

*=Anfang April bis Ende November

unter dem tiefsten Streifpunkt der Rotorblattspitzen installiert, um Membranschäden am Mikrofon durch Druckwellen der vorbeistreichenden Rotoren zu verhindern. Zudem wird damit dem über den direkten Rotorradius hinausreichenden Effekt des Barotraumas (Baerwald et al. 2008; Grodsky et al. 2011) Rechnung getragen, wenngleich hier ein genauer Wirkungsbereich in Metern nicht bekannt ist. Barotrauma können durch Unterdruckwirbel an der Spitze der Rotorblätter entstehen und zu Schäden an Lunge und Innenohr führen, was den Tod der Tiere nach sich zieht.

Alle Einstellungen des Avisoft-Recorders wurden nach den Vorgaben von RENEBAT vorgenommen und die Mikrofone auf $37 \mathrm{~dB}$ SPL kalibriert (Behr et al. 2015; Specht 2017). Die Empfindlichkeit der Gondelmikrofone wurde über einen eingebauten Signalgeber getestet und bei den Kontrollen überprüft. Die Turmmikrofone konnten technisch bedingt zwar kalibriert, aber nicht überprüft werden, wurden aber einmal im August bzw. bei erkennbaren Funktionsstörungen ausgetauscht. Die Erfassungsreichweite für Rauhautfledermäuse lag somit bei etwa 24 m, für den Abendsegler betrug sie etwa 59 m (Weber et al. 2018).

Insgesamt wurden 20 WEA jeweils von Anfang April bis Ende November untersucht, von denen je eine WEA aus dem Jahr 2017 (WEA G1) und aus dem Jahr 2018 (WEA T4) nicht berücksichtigt wurden, da es hier zu vermehrten Ausfällen wegen starker technischer Störungen kam (Tab. 5.1), sodass die Daten aus den beiden Erfassungsebenen nicht verglichen werden konnten. Von der WEA W1 wurde aus gleichen Gründen nur der Zeitraum ab dem 17.8.2018 berücksichtigt. Untersucht wurden verschiedene WEA-Typen der Firma Enercon mit Nabenhöhen von 113,5 und 135,4 m und Rotorradien von 35 und $58 \mathrm{~m}$ (Tab. 5.1). An keiner der untersuchten WEA überlagerte sich der geschätzte Erfassungsradius für Rauhautfledermäuse des Gondelmikrofons mit demjenigen des Turmmikrofons. Insgesamt wurden 29 ,WEA-Jahre“ untersucht. 
Um einen direkten Vergleich mit anderen Untersuchungen zu gewährleisten, in welchen möglicherweise andere Systeme zur Anwendung kommen, wurde die akustische Aktivität von Fledermäusen in 1-min-Intervallen berechnet. Dies bedeutet, dass je 1-min-Intervall nur jeweils eine Rufsequenz/Art zugeordnet wird, unabhängig von der Gesamtzahl der Rufsequenzen der jeweiligen Art in der entsprechenden Minute. Sind auf einer Rufdatei mehr als ein Tier einer Art zu erkennen, wurde dies als zwei besetzte 1-min-Intervalle bzw. als zwei Aktivitäten gewertet.

Alle Rufsequenzen wurden manuell, d. h. ohne Verwendung eines automatischen Identifizierungsprogramms, bestimmt. Die Bestimmung der Fledermausarten nach Rufcharakteristika richtete sich dabei nach Skiba (2007) und Barataud (2012).

\subsubsection{Statistik}

Da die untersuchten Datensätze weder normalverteilt (Shapiro-Wilk-Test) noch varianzhomogen (Unifaktorielle Varianzanalyse und Levene-Test) sind, wurden für die Signifikanztests die entsprechenden parameterfreien Tests durchgeführt:

- Kruskal-Wallis-Test mit Berechnung der Effektstärke nach Rasch und multiplen Conover-Iman-Vergleichen mit Bonferroni-Holm-Korrektur für die als unabhängige Daten betrachteten Monats-, Jahreszeiten- und Windverteilungsaktivitäten,

- Mann-Whitney-U-Test mit Berechnung der Effektstärke nach Rosenthal für den Vergleich von Turmmikrofon und Gondelhöhe

- Wilcoxon-Vorzeichen-Rang-Test mit Rosenthal-Effektstärke für die abhängigen Daten des Vergleichs der besetzten Minutenintervalle

Die Aktivitätsverteilungen wurden mittels der Unifaktoriellen Varianzanalyse (ANOVA) auf Gleichheit ihrer Varianzen überprüft und dazu Cohens Effektstärke berechnet. Die Effektstärken stehen für die praktische Relevanz von statistisch signifikanten Ergebnissen. Sie werden testspezifisch in drei Klassen unterteilt: geringer $(>)$, mittlerer $(>>)$ und großer Effekt $(>>>)$. Die statistische Auswertung wurde mit der Software BiAS für Windows in der Version 11.10-7/2019 (Ackermann 2019) durchgeführt. Die dargestellten Boxplots sind Quartilsverteilungen. Die Ermittlung der Verteilung der Nachtaktivitäten basiert auf einer Tabelle der Sonnenauf- und -untergänge für das Jahr 2017, berechnet mit dem SunEarth.Tools. com für einen Punkt im Landkreis Aurich und Wittmund (53.6336 N 7.5453 Ost). Die jährlichen sowie örtlichen Abweichungen betragen nur wenige Sekunden bis Minuten. Die Jahreszeitenauswertung bezieht sich auf die astronomischen Jahreszeiten (Tag + Uhrzeit MEZ). 


\subsection{Ergebnisse}

Insgesamt konnten an allen WEA sieben Arten plus die Gattung Plecotus (höchstwahrscheinlich das Braune Langohr [Plecotus auritus]) und nicht näher bestimmbare Tiere der Gruppe Nyctaloid (Großer Abendsegler [Nyctalus noctula], Kleinabendsegler [Nyctalus leisleri], Zweifarbfledermaus [Vespertilio murinus], Breitflügelfledermaus [Eptesicus serotinus]) sicher nachgewiesen werden (Tab. 5.2).

Tab.5.2 Gesamtübersicht der Fledermausaktivität in Minutenintervallen auf Gondelhöhe und am Turmmikrofon ( $\mathrm{n}=$ Anzahl „WEA-Jahre“)

Tab.5.2 Overview of the bat activity in 1-min-intervalls at nacelle heigth and at the tower microphone ( $\mathrm{n}=$ number of ,wind turbine-years")

\begin{tabular}{l|l|l|l|l|l|l}
\hline Windpark Gondelhöhe & $\mathrm{H}$ & $\mathrm{T}$ & $\mathrm{S}$ & $\mathrm{G}$ & $\mathrm{U}$ & $\mathrm{W}$ \\
\hline WEA-Jahre & $\mathbf{2}$ & $\mathbf{5}$ & $\mathbf{1 0}$ & $\mathbf{7}$ & $\mathbf{3}$ & $\mathbf{2}$ \\
\hline Abendsegler (Nyctalus noctula) & 195 & 230 & 532 & 64 & 124 & 261 \\
\hline Kleinabendsegler (Nyctalus leisleri) & 3 & 15 & 7 & & 3 & \\
\hline Breitflügelfledermaus (Eptesicus serotinus) & & 7 & 20 & 1 & & 1 \\
\hline Zweifarbfledermaus (Vespertilio murinus) & & & & & & \\
\hline Nyctaloid & 172 & 26 & 21 & 1 & & 178 \\
\hline Rauhautfledermaus (Pipistrellus nathusii) & 238 & 95 & 236 & 12 & 22 & 189 \\
\hline Zwergfledermaus (Pipistrellus pipistrellus) & 7 & 1 & 2 & & & 2 \\
\hline Pipistrellus spec & 2 & & & & & \\
\hline Langohr (Plecotus spec.) & 6 & & 2 & & & 1 \\
\hline Windpark Turmmikrofon & $\mathrm{H}$ & $\mathrm{T}$ & $\mathrm{S}$ & $\mathrm{G}$ & $\mathrm{U}$ & $\mathrm{W}$ \\
\hline WEA-Jahre & $\mathbf{2}$ & $\mathbf{5}$ & $\mathbf{1 0}$ & $\mathbf{7}$ & $\mathbf{3}$ & $\mathbf{2}$ \\
\hline Abendsegler (Nyctalus noctula) & 160 & 355 & 1732 & 563 & 608 & 709 \\
\hline Kleinabendsegler (Nyctalus leisleri) & 1 & 6 & 16 & & 13 & \\
\hline Breitflügelfledermaus (Eptesicus serotinus) & & 21 & 124 & 20 & 61 & 11 \\
\hline Zweifarbfledermaus (Vespertilio murinus) & 1 & 1 & & & & \\
\hline Nyctaloid & 229 & 49 & 40 & 9 & 49 & 921 \\
\hline Rauhautfledermaus (Pipistrellus nathusii) & 375 & 330 & 789 & 178 & 246 & 604 \\
\hline Zwergfledermaus (Pipistrellus pipistrellus) & 10 & 2 & 1 & 2 & 2 & 32 \\
\hline Pipistrellus spec & 1 & & & & & \\
\hline Teichfledermaus (Myotis dasycneme) & & & 2 & & & \\
\hline Langohr (Plecotus spec.) & 11 & 2 & 2 & 2 & 8 & 7 \\
\hline
\end{tabular}




\subsubsection{Unterschiede der akustischen Aktivität aller Fledermausarten zwischen Gondel- und Turmmikrofon}

An allen WEA, bis auf den Standort T3 im Windpark T im Jahr 2018, lag die Gesamtaktivität aller Fledermausarten (ohne Rauhautfledermäuse) an den Turmmikrofonen höher als an den Gondelmikrofonen. Der Median des faktoriellen Unterschieds lag bei 3,1. Die Zahl der Aktivitäten im Bereich der unteren Rotorspitze lag um das 1,1- bis 215-Fache und im Median 3,1-fach so hoch wie auf Nabenhöhe. Eine Ausnahme stellten WEA T3 und S3 aus dem Jahr 2018 dar, wo im Gegensatz zum generellen Muster die akustische Aktivität der Fledermäuse am Gondelmikrofon höher war als am Turmmikrofon.

Neben zum Teil deutlichen Schwankungen zwischen den Jahren (z. B. WEA H) zeigten sich auch Unterschiede zwischen den einzelnen WEA, und zwar in der allgemeinen akustischen Gesamtaktivität sowohl am Gondel- als auch am Turmmikrofon sowie im Verhältnis der Aktivität an den beiden Mikrofonstandorten zueinander (Abb. 5.1).

Zusätzlich zur allgemeinen höheren Gesamtaktivität von Fledermäusen am Turmmikrofon konnten an den meisten beprobten WEA auf Höhe des Turmmikrofons bei 20 von 29 WEA auch weitere Arten festgestellt werden, die mit dem Gondelmikrofon nicht erfasst wurden. Dies betraf vor allem Breitflügelfledermäuse (Eptesicus

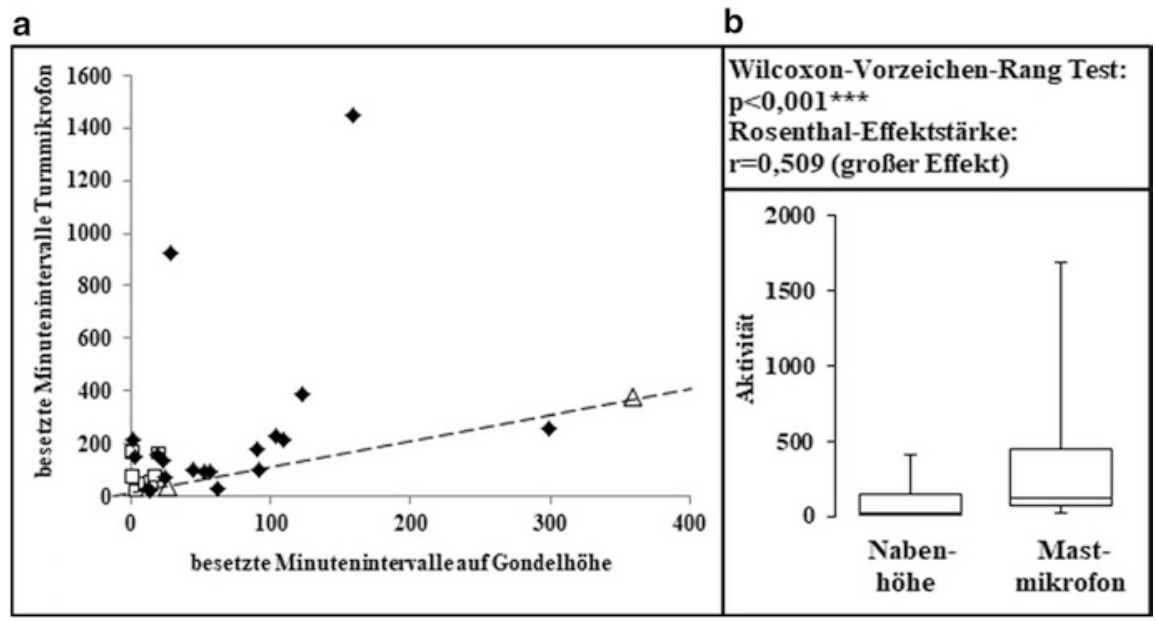

Abb. 5.1 Akustische Gesamtaktivität ohne Rauhautfledermaus in den 29 beprobten WEAJahren. Dreieck=Enercon E70, Quadrat=Enercon E101, Raute =Enercon E115; gestrichelte Linie $=1: 1$-Linie

Fig. 5.1 Acoustical total activity without Nathusius' pipistrelles at the studied wind turbines. Triangle $=$ Enercon E70, quadrat $=$ Enercon E101, homb $=$ Enercon E115; dashed line $=$ line of equivalence 
serotinus), die Zwergfledermäuse (Pipistrellus pipistrellus) und Langohren (Plecotus spec.), die zwar am Turmmikrofon, nicht jedoch mit dem Gondelmikrofon dokumentiert wurden (Tab. 5.2).

\subsubsection{Unterschiede der akustischen Aktivität von Rauhautfledermäusen zwischen Gondel- und Turmmikrofon}

Betrachtet man nur die akustische Aktivität der Rauhautfledermäuse (Abb. 5.2), so zeigte sich ein ähnliches Bild wie bei der Gesamtaktivität. Auch hier ließen sich standortspezifische Unterschiede registrieren. Der Median des faktoriellen Unterschieds lag bei 4,2. Die Zahl der Aktivitäten im Bereich der unteren Rotorspitze lag um das 1,5- bis 77-Fache so hoch wie auf Nabenhöhe. Stark ausgeprägt waren die Unterschiede zwischen Gondel- und Turmmikrofon an den Standorten S5 im Jahr 2017, W2 und U1 im Jahr 2018 (Abb. 5.2).

Dabei ist nicht nur die generell höhere Aktivität der Rauhautfledermaus im Bereich der unteren Rotorspitze entscheidend, sondern auch die unterschiedliche saisonale und nächtliche Verteilung. So ist die akustische Aktivität am Turmmikrofon in der Regel nicht nur in den gleichen Nächten höher als am Gondelmikrofon, sondern sie verteilt sich auch saisonal unterschiedlich. Die Aktivität am Turmmikrofon ist im August und September bis in den Oktober hinein signifikant höher

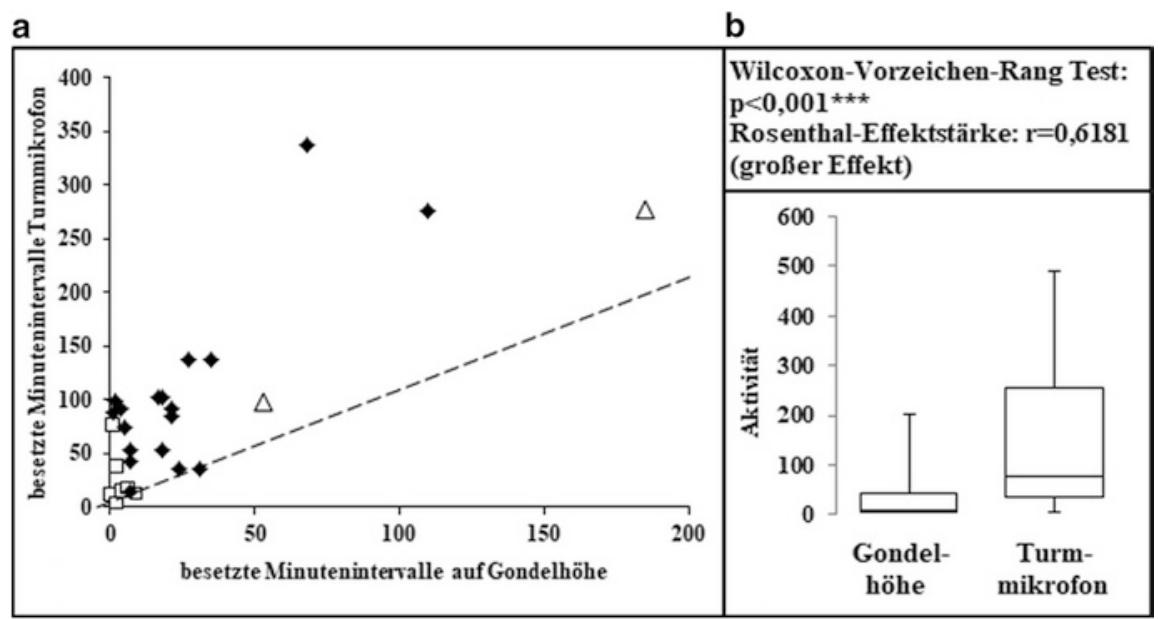

Abb. 5.2 Akustische Aktivität der Rauhautfledermaus an den 29 beprobten WEA. Dreieck $=$ Enercon 70, Quadrat $=$ Enercon 101, Raute $=$ Enercon 115; gestrichelte Linie = 1:1-Linie

Fig. 5.2 Acoustic activity of Nathusius' pipistrelles at the studied wind turbines. Triangle $=$ Enercon E70, quadrat $=$ Enercon E101, rhomb $=$ Enercon E115; dashed line $=$ line of equivalence 


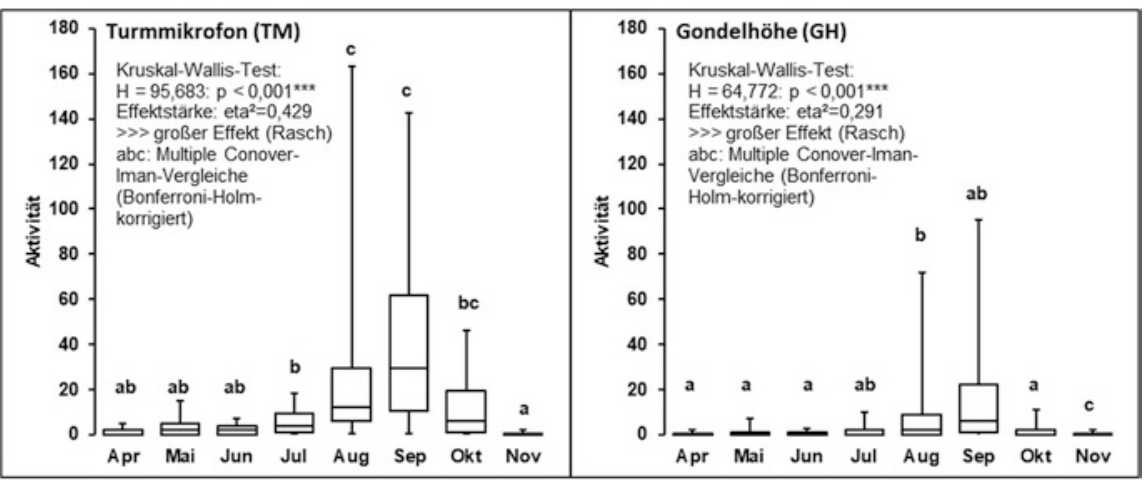

Abb. 5.3 Aktivitätsverteilung der Rauhautfledermaus an den 29 beprobten WEA in den Monaten April bis November am Turmmikrofon (links) und in Gondelhöhe (rechts)

Fig. 5.3 Distribution of Nathusius' pipistrelles activity at the tower microphone (left) and at nacelle height (right) shown at all wind turbines from April until November

als in den anderen Monaten, während sie auf Gondelhöhe im Juli bis September signifikant, jedoch mit schwächerem Effekt ausfällt (Abb. 5.3). Wichtig dabei ist neben der generell und signifikant höheren Gesamtaktivität am Turmmikrofon vor allem das sich über die Saison verändernde Verhältnis der Aktivität von Turmmikrofon und Gondelhöhe (Abb. 5.4). Die Aktivitäten am Turmmikrofon und in Gondelhöhe unterscheiden sich zudem bzgl. der Monatsverteilung signifikant $($ ANOVA $\mathrm{p}<0,001 * * *=$ nicht varianzhomogen; Cohens Effektstärke $=0,529>>$ mittlerer Effekt).

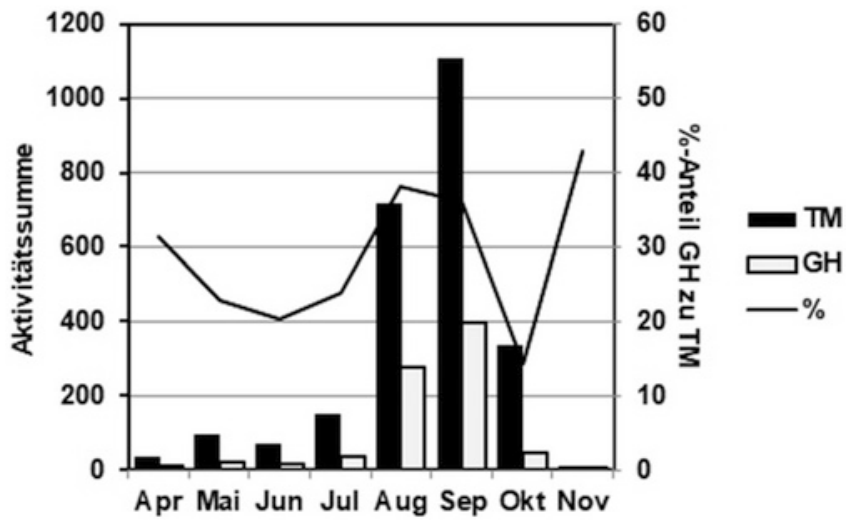

Abb. 5.4 Verteilung der Gesamtaktivität der Rauhautfledermaus als Summe der beprobten WEA in den Monaten April bis November. $\mathrm{TM}=$ Turmmikrofon, $\mathrm{GH}=$ Gondelhöhe

Fig. 5.4 Distribution of Nathusius' pipistrelles activity as sum of all wind turbines from April until November. TM= tower microphone, $\mathrm{GH}=$ nacelle height 


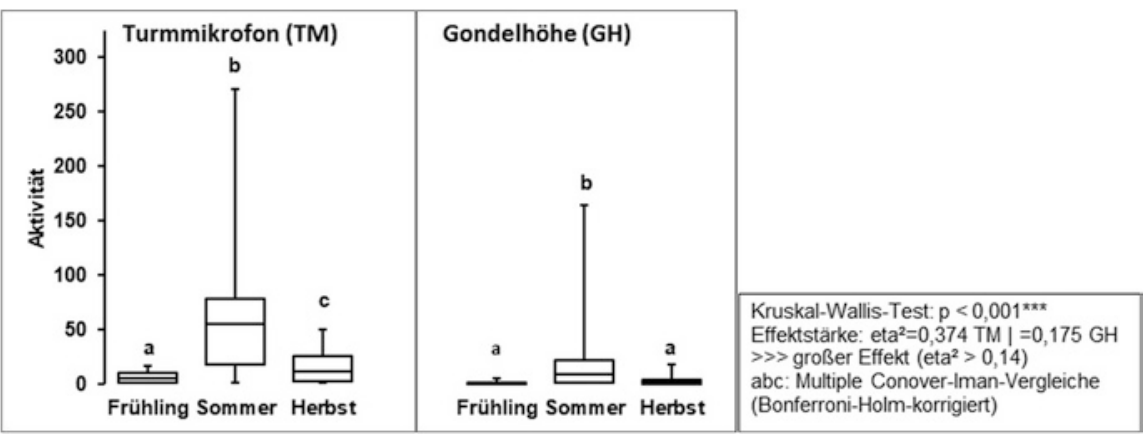

Abb. 5.5 Jahreszeitliche Aktivitätsverteilung der Rauhautfledermaus an den beprobten WEA.

Fig. 5.5 Seasonal distribution of Nathusius' pipistrelles activity at all sampled wind turbines

Eine ähnliche Aktivitätsverteilung zeigt sich auch in den Jahreszeiten (Abb. 5.5). Die Aktivität ist sowohl am Turmmikrofon als auch in Gondelhöhe im Sommer signifikant höher als im Frühjahr und Herbst, wobei am Turmmikrofon auch noch im Herbst signifikant höhere Aktivitäten als im Frühjahr erfasst wurden. Auch hier unterscheiden sich die Aktivitätsverteilungen signifikant (ANOVA $\mathrm{p}<0,001 * * *$, Cohens Effektstärke $=1,467>>>$ großer Effekt).

Ein ebenfalls ähnliches Ergebnis zeigt die nächtliche Verteilung der Aktivität, wobei die Nacht wegen der Vergleichbarkeit in Nacht-Zehntel aufgeteilt ist (Abb. 5.6). Zwar ist an beiden Mikrofonen die höchste Aktivität etwa im dritten und vierten Zehntel nach Sonnenuntergang zu verzeichnen und nimmt nachfolgend stetig ab, doch nimmt die Aktivität auf Gondelhöhe im Vergleich zum

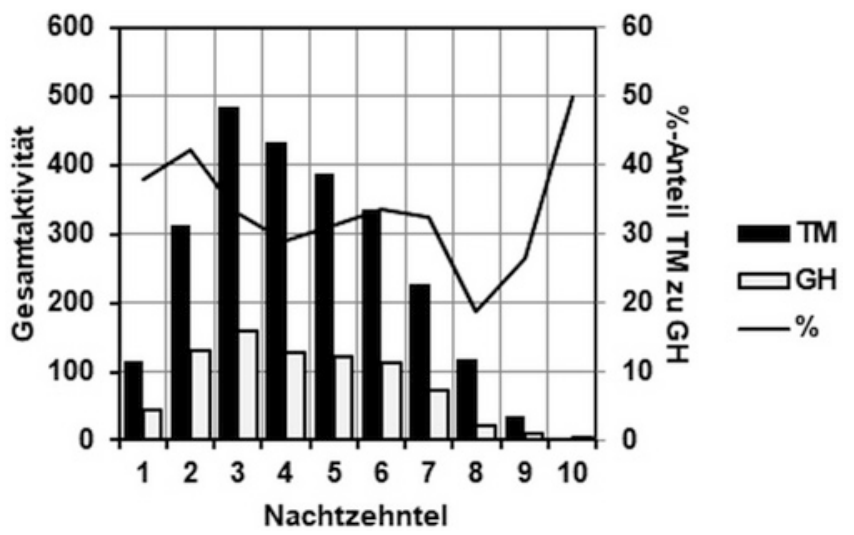

Abb. 5.6 Nächtliche Aktivitätsverteilung der Rauhautfledermaus als Summe aller beprobten WEA. TM=Turmmikrofon, $\mathrm{GH}=$ Gondelhöhe; Nacht aufgeteilt in Zehntelphasen

Fig. 5.6 Nocturnal distribution of Nathusius' pipistrelles activity shown as sum of all sampled wind turbines. $\mathrm{TM}=$ tower microphone, $\mathrm{GH}=$ nacelle height; the night divided in deciles 
Turmmikrofon im Laufe der Nacht prozentual bis zum achten Zehntel hin deutlich stärker ab (von ca. 40 auf ca. $20 \%$ ). Die Aktivitätsverteilungen am Turmmikrofon und in Gondelhöhe unterscheiden sich auch bzgl. der Nachtverteilung signifikant (ANOVA p $<0,01 * *$, Cohens Effektstärke $=1,29>>>$ großer Effekt).

Neben der unterschiedlichen monatlichen, saisonalen und nächtlichen Verteilung der Aktivität zeigt sich auch eine unterschiedliche witterungsbedingte Nutzung, vor allem für Wind (Abb. 5.7). Während sich die Aktivitätsverteilung bezüglich Temperatur nur gering unterscheidet (maximale Aktivität in beiden Fällen bei etwa $16{ }^{\circ} \mathrm{C}$, gemessen oberhalb der Gondel!), ergibt sich für das Turmmikrofon eine statistisch signifikante Bevorzugung der Windklassen 6 und 7 (Maximum der Aktivität bei 5,5 m/s), während auf Nabenhöhe eine Bevorzugung der Windklassen 5 bis 8 mit Präferenz bei 7 vorliegt (Maximum der Aktivität bei $7 \mathrm{~m} / \mathrm{s}$ ). Wenngleich das Maximum am Turmmikrofon bei niedrigeren Windgeschwindigkeiten liegt, zeigt sich aber infolge der höheren Aktivität, dass trotzdem

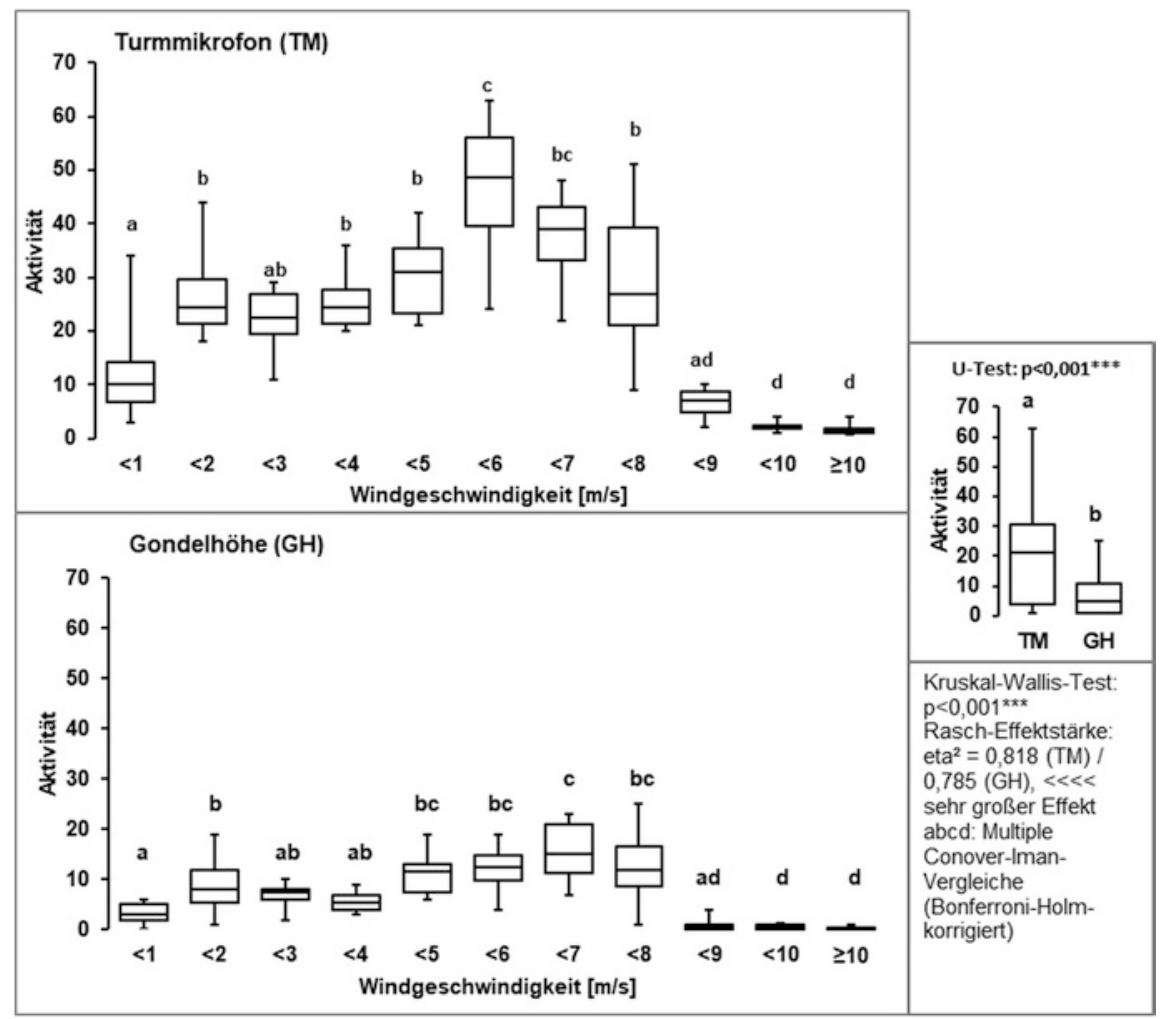

Abb. 5.7 Windabhängige Aktivitätsverteilung der Rauhautfledermaus an den 29 beprobten WEA. $\mathrm{TM}=$ Turmmikrofon, $\mathrm{GH}=$ Gondelhöhe

Fig. 5.7 Wind-dependent distribution of Nathusius' pipistrelles activity at all wind turbines. $\mathrm{TM}=$ tower microphone, $\mathrm{GH}=$ nacelle height 
noch etwa 4,5\% der Rauhautfledermausaktivität (109 besetzte Minutenintervalle) am Turmmikrofon bei auf Nabenhöhe gemessenen Windgeschwindigkeiten $\geq 8,0 \mathrm{~m} / \mathrm{s}$ auftraten, wogegen dies nur bei 2,1\% (16 besetzte Minutenintervalle) auf Nabenhöhe der Fall war. Die Aktivitätsverteilungen am Turmmikrofon und in Gondelhöhe unterscheiden sich auch bzgl. der Windverteilung signifikant (ANOVA p $<0,001 * * *$, Cohens Effektstärke = 1,13 >>> großer Effekt).

\subsection{Diskussion}

Frühere Studien zeigten, dass prinzipiell die akustische Aktivität von Fledermäusen in Gondelhöhe und Schlagraten an den jeweiligen WEA korrelierten (Brinkmann et al. 2011; Roemer et al. 2017). Allerdings variierte die Qualität dieser Korrelation standortabhängig (Brinkmann et al. 2011; Rydell et al. 2010; Behr et al. 2015, 2018; NATURA \& SWILD 2018). Die Nutzung der akustischen Aktivität in Gondelhöhe schien deshalb ausreichend gut zu sein, um das Kollisionsrisiko an der jeweiligen WEA abschätzen zu können. Neuere Veröffentlichungen nähren jedoch Zweifel, ob die Übertragbarkeit, speziell der Berechnungsmethode nach ProBat bei größeren Anlagen möglich ist (Lindemann et al. 2018). Monitoringuntersuchungen an 59 WEA (90 WEA-Jahre) zeigen vor allem an küstennahen Standorten, dass die akustische Aktivität der Rauhautfledermaus in Gondelhöhe und der Schlagopferzahl unter der jeweiligen WEA mitunter nicht signifikant korreliert (Kap.4). Zu ähnlichen Ergebnissen betreffend die Gattung Pipistrellus kommen NATURA \& SWILD (2018) in der küstenfernen Schweiz an einem Standort mit drei WEA mit vergleichsweise geringer Gesamtaktivität aber relativ hohem Anteil an Pipistrellen an der Aktivität. Für die Ermittlung eines fledermausfreundlichen Betriebs der WEA ist die behördliche Festlegung eines Grenzwertes für die Schlagopferzahl je WEA notwendig. Die Festlegung eines kritischen Grenzwertes wiederum bedeutet jedoch, dass die Ermittlung der Schlagrate entsprechend exakt erfolgen muss. Dies kann durch eine möglichst tägliche Schlagopfersuche oder durch die Korrelation von akustischer Aktivität in Gondelhöhe und geschätzter Schlagrate erfolgen. Hier bieten sich die Erfahrungen und die Berechnungsmethode nach ProBat aus den RENEBAT-Projekten an. Vor allem die neue ProBat-Version 6.1 geht z. B. speziell auf die Rauhautfledermaus und die Regionalisierung (z. B. Küstenstandorte) ein. ProBat besitzt den Vorteil, dass Abschaltalgorithmen objektiv ermittelt werden. Dies gilt allerdings nur, wenn die Erfassungen und die planerische Umsetzung über die Empfehlungen von RENEBAT bzw. ProBat (Ermittlung des Abschaltalgorithmus) in adäquater Weise erfolgen.

Hierbei bestehen momentan noch diverse Probleme, wie beispielsweise die bedingte Übertragbarkeit des ursprünglichen Datensatzes auf WEA mit größeren Rotorlängen, welche neben dem steigenden Wirkradius der Rotoren (durchstrichener Luftraum) auch ein relativ zum Wirkradius der Rotoren reduzierter 
Erfassungsraum der einzelnen Fledermausarten bedeutet (Lindemann et al. 2018). Dies könnte erklären, dass mitunter trotz geringer akustischer Aktivität von Fledermäusen gemessen auf Nabenhöhe regelmäßig Schlagopfer auftreten ein Problem, das bei relativ hochfrequent rufenden Arten mit daraus folgender geringerer Erfassungsreichweite durch Ultraschallmikrofone, wie zum Beispiel bei Rauhautfledermäusen, massiver auftritt.

Rauhautfledermäuse fallen als Schlagopfer an WEA schon bei relativ geringer akustischer Aktivität auf Nabenhöhe an. So wurde in küstennahen Windparks durchschnittlich bei 57 akustischen Aktivitätsereignissen eine tote Rauhautfledermaus gefunden (Kap.4). Unter Berücksichtigung der Abtragsrate, der Sucheffizienz und der absuchbaren Fläche (Berechnung nach Brinkmann et al. 2006 bzw. Korner-Nievergelt et al. 2011) würde ein Schlagopfer pro 27 Aktivitätsereignisse auftreten. Betrachtet man nur den Zeitraum von August bis September, den Zeitraum mit der höchsten Schlagwahrscheinlichkeit (Arnett et al. 2008, Brinkmann et al. 2011, Rodrigues et al. 2015), sinkt das Verhältnis auf ein Schlagopfer pro 25 Aktivitätsereignisse für Rauhautfledermäuse (Kap. 4). Auch NATURA \& SWILD (2018) fanden in der Schweiz, dass schon geringe, auf Nabenhöhe erfasste Aktivitäten der Gattung Pipistrellus zu Schlagopfern führten. In ihrem Falle betraf es aber nicht nur Rauhautfledermäuse, sondern auch Zwergfledermäuse.

Die mit zunehmender Höhe abnehmende Aktivität betrifft in der Regel alle Arten bzw. Artengruppen. Unsere Studie zeigt, dass beispielsweise im Bereich der unteren Rotorspitze vermehrt Zwerg- und Breitflügelfledermäuse detektiert werden konnten. Auch Langohren und Mausohrartige (z. B. Teichfledermaus) wurden dort immer wieder erfasst. Hier ist allerdings infolge der Seltenheit dieser Ereignisse, zumindest bei den hier untersuchten WEA, nicht mit einem erhöhten Schlagrisiko zu rechnen. Eine mit abnehmender Höhe über dem Boden zunehmende Aktivität von Fledermäusen sowie eine höhere Artenzahl wurden bereits mehrfach beschrieben (z. B. Arnett et al. 2008; Brinkmann et al. 2011; Hurst et al. 2016; Menzel et al. 2005). Auf der anderen Seite zeigte sich an WEA T3, dass es, zumindest die Gesamtaktivität betreffend, in Ausnahmefällen auch umgekehrt sein kann. Der Grund für die höhere Aktivität auf Nabenhöhe an dieser Anlage waren Individuen des Großen Abendseglers, die auf Nabenhöhe etwa doppelt so häufig aufgenommen wurden als im Bereich der unteren Rotorspitze. Ähnliche Ergebnisse liegen aus dem nördlichen Emsland bei Rhede vor, wo auf Nabenhöhe deutlich mehr Aktivität verzeichnet werden konnte als im Bereich der unteren Rotorspitze (Deiting mündlich). Im Gegensatz zu den hier dargestellten Daten (höhere Aktivität auf Nabenhöhe nur im Falle der Gesamtaktivität!) betrifft die höhere Aktivität auf Nabenhöhe dort aber sowohl die Gesamtaktivität als auch jene der Rauhautfledermaus. Eine Erklärung hierfür ist bislang nicht möglich; der Verdacht liegt nahe, dass es sich um einen (natur-)räumlichen Effekt handeln könnte. Hier müssen weitere Ergebnisse aus anderen Regionen abgewartet werden. 
Ein Faktor, dem bislang wenig Beachtung geschenkt wurde, ist die unterschiedliche saisonale und nächtliche Verteilung der akustischen Aktivität an WEA. Dabei zeigt sich, dass sich zwar die saisonale Hauptaktivitätsphase (August, September) auf Nabenhöhe auch im Bereich der unteren Rotorspitze widerspiegelt, allerdings können auch höhere Aktivitäten an der unteren Rotorspitze auftreten, die sich nicht auf Nabenhöhe abzeichnen (z. B. Oktober). Zudem verändert sich das Verhältnis der Aktivität von Turmmikrofon zur Gondelhöhe über die Saison. Vergleichbares findet sich bei der zeitlichen Verteilung der akustischen Aktivitäten während der Nacht wieder, wo sich die Aktivitäten im Bereich der unteren Rotorspitze auf Gondelhöhe im Laufe der Nacht immer schlechter widerspiegeln. Gerade aber solche asynchronen Aktivitätsmuster im Wirkbereich der Rotoren könnten zu Fehlern beim Festlegen der Abschaltmodi führen, wenn diese alleine auf Basis der Aktivitätserfassungen auf Nabenhöhe erfolgen.

Neben der eigentlichen Aktivität spielt vor allem die Windabhängigkeit der Aktivität eine entscheidende Rolle bei der Festlegung und Beauflagung von Abschaltungen. Dies gilt sowohl für den Artenschutz (Bach \& Bach 2009; Brinkmann et al. 2011) als auch für die Wirtschaftlichkeit der WEA (www.windturbine-models.com, Stand 2019). Wie die oben dargestellten Ergebnisse zeigen, fliegen die Rauhautfledermäuse im Bereich der unteren Rotorspitze augenscheinlich noch bei höheren Windgeschwindigkeiten als auf Nabenhöhe. Dies gilt auch für die meisten übrigen Arten. Hier muss bedacht werden, dass die Windgeschwindigkeit an allen WEA oberhalb der Gondel gemessen wurde, die akustische Aktivität aber am Turm unterhalb der unteren Rotorspitze ermittelt wird. Der Wind nimmt im Allgemeinen mit abnehmender Höhe über dem Boden ab (Lindemann et al. 2018), was zur Folge hat, dass die tatsächlichen Windgeschwindigkeiten im Erfassungsbereich der Mikrofone (ca. 20-40 m Distanz zum Mikrofon) nicht jenen auf dem Dach der Gondel gemessenen Werten entsprechen. Rauhautfledermäuse fliegen also im Bereich der unteren Rotorspitze i. d. R. bei geringerer Windgeschwindigkeit als jener, die gleichzeitig auf Nabenhöhe gemessen wird. Demzufolge darf aus den Aktivitätsdaten im Bereich der unteren Rotorspitze bei scheinbar hohen Windgeschwindigkeiten nicht auf eine höhere Windtoleranz der Tiere geschlossen werden. Für die Ermittlung des Abschaltmodus ist dies allerdings irrelevant, da er anschließend aus den Werten der auf Nabenhöhe gemessenen Werte gesteuert wird.

Die Ergebnisse unserer Studie zeigen, dass der Betrieb eines zweiten Mikrofons etwa im Bereich der unteren Rotorspitze ein anderes saisonales und nächtliches Aktivitätsmuster zeigt als bei akustischen Aufnahmen auf Nabenhöhe. Damit ergänzen die Daten eines Turmmikrofons die Ergebnisse der akustischen Erfassung auf Nabenhöhe. Diese Daten sollten ein besseres Bild über die Aktivitäten im Wirkbereich des Rotors ermöglichen und speziell das Auftreten von Schlagopfern der Rauhautfledermaus bei keinen oder extrem wenigen auf Nabenhöhe erfassten Aktivitäten erklären helfen. Dabei stellen sich allerdings 
drei Probleme. Zum einen werden mit einem waagerecht ausgerichteten Mikrofon im Bereich der unteren Rotorspitze Aktivitäten der Rauhautfledermaus (und aller anderen Arten) aufgenommen, welche ggf. nicht im Wirkradius des Rotors fliegen. Das zweite, bislang nicht gelöste Problem ist, dass keine Untersuchungen existieren, welche eine Korrelation der Schlagrate zu der oben dargestellten Kombination der Aktivitätserfassung erlauben. Das dritte Problem bezieht sich auf die Anbringung des Turmmikrofons. Im Gegensatz zum Gondelmikrofon, welches sich mit der Gondel windabhängig dreht, ist das Turmmikrofon fixiert - üblicherweise an der Stelle, an der im Turm die Leiter befestigt ist, was zwar je Anlagentyp festgelegt ist, sich am jeweiligen Standort aber durchaus in der Himmelsrichtung unterscheiden kann. Hier wäre dauerhaft eine festzulegende Himmelsrichtung anzustreben. Auch betreffend die wettergeschützte Anbringung des Mikrofons sind noch Verbesserungen möglich. Ein weiteres Problem der Anbringung des zweiten Mikrofons ist die Anbringung unterhalb der unteren Rotorspitze. Hier wurde immer wieder die Kritik geäußert, dass Tiere aufgenommen werden, die nicht im Rotorwirkbereich fliegen und damit gar nicht schlaggefährdet sind. Es sollte daher dringend geprüft werden, ob eine Anbringung des zweiten Mikrofons wenige Meter oberhalb der unteren Rotorspitze machbar ist.

\subsection{Fazit}

Die Daten des Turmmikrofons auf Höhe des unteren Streifpunkts der Rotorspitzen können die akustischen Aufnahmen auf Nabenhöhe einer WEA ergänzen und stellen zumindest für Küstenstandorte und eventuell auch für Waldstandorte ein realistischeres Bild der Aktivitäten im Wirkbereich des Rotors dar. Aus diesem Grund ist die zusätzliche Installation eines zweiten Mikrofons im Bereich der unteren Rotorspitze sinnvoll und sollte in Landschaften mit verstärktem Auftreten von Rauhautfledermäusen (z. B. Nordwestdeutschland) und bei Rotorlängen, welche die Erfassungsreichweite von Rauhautfledermäusen übertreffen, vorangetrieben werden. Die fehlende Synchronität der erfassten Aktivitäten und die Erfassungsreichweite der Mikrofone belegen auch, dass es nicht sinnvoll ist, die Erfassung auf Nabenhöhe ersatzlos durch ein Turmmikrofon zu ersetzen. Im Gegenteil: Die bisherigen Erfahrungen und statistischen Berechnungen (Brinkmann et al. 2011; Behr et al. 2015, 2018) sollten genutzt und mit Daten eines zweiten Mikrofons ergänzt und in die Errechnung des Abschaltalgorithmus integriert werden. Um die Akustikdaten in das Berechnungssystem von ProBat einzupflegen, müsste dieses entsprechend angepasst werden, was weitere Forschung in diese Richtung erfordern würde. Eine realistische und fundierte Berechnung ist umso wichtiger, wenn Abschaltungen an WEA vorgenommen werden sollen, wo keine Überprüfungen von auftretenden Schlagopfern möglich sind, z. B. offshore (Mollis et al. 2019). 
Danksagung An dieser Stelle sei all jenen Personen gedankt, welche die obigen Untersuchungen ermöglicht haben. Hier sind besonders Herr Poppen (Landkreis Aurich) und Frau Kuklok-Grimm und Herr Frerichs (Landkreis Wittmund) zu nennen. Außerdem wurden die Ergebnisse intensiv mit folgenden Personen diskutiert: Oliver Behr, Carsten Dense, Johannes Deiting, Kerstin Frey, Anna Lotter, Gerd Mäscher, Sarah Middeljans, Theodor Poppen und Christian Voigt. Nicht zuletzt gilt unser Dank all jenen Betreibern, die uns die Veröffentlichung der Monitoringdaten erlaubt haben.

Die Veröffentlichung wurde durch den Open-Access-Publikationsfonds für Monografien der Leibniz-Gemeinschaft gefördert.

\section{Literatur}

Ackermann H (2019) BiAS. für Windows. Biometrische Analyse von Stichproben. Handbuch Version 11. EPSiLON Verlag, Hochheim, S 247

Arnett EB, May RF (2016) Mitigation wind energy impacts on wildlife: approaches for multiple taxa. Human-Wildlife Interactions 10:28-41

Arnett E, Brown WK, Erickson WP, Fiedler JK, Hamilton BL, Henry TH, Jain A, Johnson GD, Kerns J, Koford RR, Nicholson CP, O’Connel TJ, Piorkowski MS, Tankersley RD (2008) Patterns of bat fatalities at wind energy facilities in North America. J Wildl Manag 72:61-78

Bach L, Bach P (2009) Einfluss der Windgeschwindigkeit auf die Aktivität von Fledermäusen. Nyctalus 14:3-13

Baerwald EF, D'Amours GH, Klug BJ, Barclay RMR (2008) Barotrauma is a significant cause of bat fatalities at wind turbines. Current Biol 18:695-696

Barataud M (2012) Acoustic Ecology of European Bats. Biotope Édition Publications scientifique du Muséum, Paris, S 348

Behr O, Brinkmann R, Korner-Nievergelt F, Nagy M, Niermann I, Reich M, Simon R (2015) Reducing the collision risk for bats at onshore wind turbines (RENEBAT II) - Summary. Leibniz Universität Hannover, Hannover

Behr O, Brinkmann R, Hochradel K, Mages J, Korner-Nievergelt F, Reinhard H, Simon R, Stiller F, Weber N, Nagy M (2018). Bestimmung des Kollisionsrisikos von Fledermäusen an Onshore-Windenergieanlagen in der Planungspraxis - Endbericht des Forschungsvorhabens gefördert durch das Bundesministerium für Wirtschaft und Energie (Förderkennzeichen 0327638E). In: Behr O. et al., Erlangen, Freiburg, Ettiswil

Bernardino J, Bispo H, Mascarenhas M (2013) Estimating bird and bat mortalities at wind farms: a practical overview of estimators, their assumptions and limitations. N Z J Zool 40:63-74

Brinkmann R, Schauer-Weisshahn H, Bontadina F (2006) Untersuchungen zu möglichen betriebsbedingten Auswirkungen von Windkraftanlagen auf Fledermäuse im Regierungsbezirk Freiburg. Unveröff. Gutachten für das Regierungspräsidium, S 66

Brinkmann R, Behr O, Niermann I, Reich M (2011) Entwicklung von Methoden zur Untersuchung und Reduktion des Kollisionsrisikos von Fledermäusen an Onshore-Windenergieanlagen. Umwelt und Raum Bd. 4, Cuvillier Verlag, Göttingen BWE (2018). https://www.wind-energie.de/fileadmin/_processed_/4/8/csm_Factsheet_-_Windenergie_in_Deutschland_2018_d3ab9e66ea.jpg

BWE (Bundesverband Windenergie) (Juni 2018) https://www.wind-energie.de/

Corten GP, Veldkamp HF (2001) Insects can halve wind-turbine power. Nature 412:42-43

Frick WF, Baerwald EF, Pollock JF, Barclay RMR, Szymanski JA, Weller TJ, Russel AL, Loeb SC, Medellin RA, McGuire LMP (2017) Fatalities at wind turbines may threaten population viability of a migratory bat. Biol Conserv 209:172-177

Grodsky SM, Behr MJ, Gendler A, Drake D, Dieterle BD, Rudd RJ, Walrath NL (2011) Investigating the causes of death for wind turbine-associated bat fatalities. J Mammal 92:917-925 
Hurst J, Biedermann M, Dietz M, Krannich E, Karst I, Korner-Nievergelt F, Schauer-Weisshahn H, Schorcht W, Brinkmann R (2016) Fledermausaktivität in verschiedenen Höhen über dem Wald. In: Hurst J, Biedermann M, Dietz C, Dietz M, Karst I, Krannich E, Petermann R, Schorcht W, Brinkmann R (Hrsg) Fledermäuse und Windkraft im Wald. Bundesamt für Naturschutz, Bonn-Bad Godesberg, S 327-352

Korner-Nievergelt F, Behr O, Niermann I, Brinkmann R (2011) Schätzung der Zahl verunglückter Fledermäuse an Windenergieanlagen mittels akustischer Aktivitätsmessungen und modifizierter N-mixture Modelle. In: Brinkmann R, Behr O, Niermann I, Reich M (Hrsg) Entwicklung von Methoden zur Untersuchung und Reduktion des Kollisionsrisikos von Fledermäusen an Onshore-Windenergieanlagen. Umwelt und Raum Bd 4. Cuvillier Verlag, Göttingen, S 323-353

Lindemann C, Runkel C, Kiefer A, Lukas A, Veith M (2018) Abschaltalgorithmen für Fledermäuse an Windenergieanlagen. Naturschutz und Landschaftsplanung 50:418-425

Menzel JM, Menzel MA, Kilgo JC, Ford WM, Edwards JW, McCracken GF (2005) Effect of habitat and foraging height on bat activity in the coastal plain of South Carolina. J Wildl Manag 69:235-245

Mollis M, Hill R, Hüppop O, Bach L, Coppack T, Pelletier S, Dittmann T, Schulz A (2019) Chapter 6: Measuring and monitoring bird collision and avoidance. In: Perrow MR (Hrsg) Wildlife and wind farms, conflicts and solutions Vol. 4 Offshore: monitoring and mitigation. Pelagic Publishing, Exeter, UK, S 167-206

NATURA \& SWILD (2018) Mortalité causée par le parc éolien du Peuchapatte sur les chauvessouris et évaluation de mesures de protection - Mortalität von Fledermäusen beim Windpark Le Peuchapatte und Evaluation von Schutzmassnahmen. Synthesebericht, NATURA, Les Reussilles \& SWILD, Zürich, S 126

NMU (Niedersächsisches Ministerium für Umwelt, Energie und Klimaschutz) (2016) Leitfaden Umsetzung des Artenschutzes bei der Planung und Genehmigung von Windenergieanlagen in Niedersachsen. Nds. Ministerialblatt Nr. 7 vom 24.2.2016:212-225. https://www.umwelt. niedersachsen.de/windenergieerlass/windenergieerlass-133444.html

O'Shea TJ, Cryan PM, Hayman DTS, Plowright RK, Streicker DG (2016) Multiple mortality events in bats: a global review. Mammal Rev 46:1-16

Rodrigues L, Bach L, Dubourg-Savage M-J, Karapandža B, Kovač D, Kervyn T, Dekker J, Kepel A, Bach P, Collins J, Harbusch C, Park K, Micevski B, Mindermann J (2015) Guidelines for consideration of bats in wind farm projects, Revision 2014. - EUROBATS Publication Series No. 6. UNEP/EUROBATS Secretariat, Bonn, S 133

Roemer C, Disca T, Coulon A, Bas Y (2017) Bat flight height monitored from wind masts predicts mortality risk from wind farms. Biol Conserv 215:116-122

Rydell J, Bach L, Dubourg-Savage M-J, Green M, Rodrigues L, Hedenström A (2010) Bat mortality at wind turbines in northwestern Europe. Acta Chiropterol 12:261-274

Specht R (2017) Anleitung zur Inbetriebnahme und Kalibrierung des WEA-Fledermausmonitoring-Systems (Version 1.3.2017): 1 Seite. www.avisoft.com/ Inbetriebnahme und Kalibrierung des WEA-Fledermausmonitoring-Systems.pdf

Skiba R (2007) Europäische Fledermäuse. Neue Brehm Bücherei, S 220

Voigt CC, Lehnert LS, Petersons G, Adorf F, Bach L (2015) Wildlife and renewable energy: German politics cross migratory bats. Eur J Wildl 61:213-219

Weber N, Nagy M, Hochradel K, Mages J, Naucke A, Schneider A, Stiller F, Behr O, Simon R (2018) Akustische Erfassung der Fledermausaktivität an Windenergieanlagen. In: Behr O et al. (Hrsg) Bestimmung des Kollisionsrisikos von Fledermäusen an Onshore-Windenergieanlagen in der Planungspraxis - Endbericht des Forschungsvorhabens gefördert durch das Bundesministerium für Wirtschaft und Energie (Förderkennzeichen 0327638E). Erlangen, S 31-58 
Open Access Dieses Kapitel wird unter der Creative Commons Namensnennung 4.0 International Lizenz (http://creativecommons.org/licenses/by/4.0/deed.de) veröffentlicht, welche die Nutzung, Vervielfältigung, Bearbeitung, Verbreitung und Wiedergabe in jeglichem Medium und Format erlaubt, sofern Sie den/die ursprünglichen Autor(en) und die Quelle ordnungsgemäß nennen, einen Link zur Creative Commons Lizenz beifügen und angeben, ob Änderungen vorgenommen wurden.

Die in diesem Kapitel enthaltenen Bilder und sonstiges Drittmaterial unterliegen ebenfalls der genannten Creative Commons Lizenz, sofern sich aus der Abbildungslegende nichts anderes ergibt. Sofern das betreffende Material nicht unter der genannten Creative Commons Lizenz steht und die betreffende Handlung nicht nach gesetzlichen Vorschriften erlaubt ist, ist für die oben aufgeführten Weiterverwendungen des Materials die Einwilligung des jeweiligen Rechteinhabers einzuholen. 